Open J. Chem., Vol. 1(2018), Issue 1, pp. 12-18

Website: https://pisrt.org/psr-press/journals/ojc/

ISSN: 2618-0758 (online) 2618-074X (Print)

http://dx.doi.org/10.30538/psrp-ojc2018.0002

\title{
ON THE MULTIPLICATIVE DEGREE BASED TOPOLOGICAL INDICES OF CIRCULANT GRAPH
}

\author{
GHULAM HUSSAIN ${ }^{1}$, SABA NOREEN, WASEEM KHAILD
}

\begin{abstract}
Topological indices are numerical numbers associated with a graph that helps to predict many properties of underlined graph. In this paper we aim to compute multiplicative topological indices of Circulant graph.

Key words and phrases: Zagreb index; Randić index; Polynomial; Degree; Graph.
\end{abstract}

\section{Introduction}

A number, polynomial or a matrix can uniquely identify a graph. A topological index is a numeric number associated to a graph which completely describes the topology of the graph, and this quantity is invariant under the isomorphism of graphs. The degree-based topological indices are derived from degrees of vertices in the graph. These indices have many correlations to chemical properties. In other words, a topological index remains invariant under graph isomorphism. The study of topological indices, based on distance in a graph, was effectively employed in 1947 in chemistry by Weiner [1]. He introduced a distance-based topological index called the "Wiener index" to correlate properties of alkenes and the structures of their molecular graphs. Recent progress in nano-technology is attracting attention to the topological indices of molecular graphs, such as nanotubes, nanocones, and fullerenes to cut short experimental labor. Since their introduction, more than 140 topological indices have been developed, and experiments reveal that these indices, in combination, determine the material properties such as melting point, boiling point, heat of formation, toxicity, toughness,

Received 09-10-2018. Revised 02-11-2018. Accepted 03-11-2018

${ }^{1}$ Corresponding Author

(c) 2018 Ghulam Hussain, Saba Noreen, Waseem Khaild. This is an open access article distributed under the Creative Commons Attribution License, which permits unrestricted use, distribution, and reproduction in any medium, provided the original work is properly cited. 
and stability [2]. These indices play a vital role in computational and theoretical aspects of chemistry in predicting material properties [3-8].

Several algebraic polynomials have useful applications in chemistry, such as the Hosoya Polynomial (also called the Wiener polynomial) [9]. It plays a vital role in determining distance-based topological indices. Among other algebraic polynomials, the M polynomial introduced recently in 2015 [10] plays the same role in determining the closed form of many degree-based topological indices. Other famous polynomials are the first Zagreb polynomial and the second Zagreb polynomial.

A graph $G$ is an ordered pair $(V, E)$, where $V$ is the set of vertices and $E$ is the set of edges. A path from a vertex $v$ to a vertex $w$ is a sequence of vertices and edges that starts from $v$ and stops at $w$. The number of edges in a path is called the length of that path. A graph is said to be connected if there is a path between any two of its vertices. The distance $d(u, v)$ between two vertices $u$ and $v$ of a connected graph $G$ is the length of a shortest path between them. Graph theory is contributing a lion's share in many areas such as chemistry, physics, pharmacy, as well as in industry [11]. We will start with some preliminary facts. The first and second multiplicative Zagreb indices [12], respectively

$$
\begin{gathered}
M Z_{1}(G)=\prod_{u \in V(G)}\left(d_{u}\right)^{2}, \\
M Z_{2}(G)=\prod_{u v \in E(G)} d_{u} \cdot d_{u},
\end{gathered}
$$

and the Narumi-Kataymana index [13]

$$
N K(G)=\prod_{u \in V(G)} d_{u} .
$$

Like the Wiener index, these types of indices are the focus of considerable research in computational chemistry [14-17]. For example, in 2011, Gutman [14] characterized the multiplicative Zagreb indices for trees and determined the unique trees that obtained maximum and minimum values for $M_{1}(G)$ and $M_{2}(G)$, respectively. Wang et al. [17] then extended Gutman's result to the following index for k-trees,

$$
W_{1}^{s}(G)=\prod_{u \in V(G)}\left(d_{u}\right)^{s} .
$$

Notice that $s=1,2$ is the Narumi-Katayama and Zagreb index, respectively. Based on the successful consideration of multiplicative Zagreb indices, Eliasi et al. [18] continued to define a new multiplicative version of the first Zagreb index as

$$
M Z_{1}^{*}(G)=\prod_{u v \in E(G)}\left(d_{u}+d_{u}\right)
$$


Furthering the concept of indexing with the edge set, the researchers introduced the first and second hyper-Zagreb indices of a graph [19] as

$$
\begin{aligned}
& H I I_{1}(G)=\prod_{u v \in E(G)}\left(d_{u}+d_{u}\right)^{2}, \\
& H I I_{2}(G)=\prod_{u v \in E(G)}\left(d_{u} \cdot d_{u}\right)^{2} .
\end{aligned}
$$

In [20], Kulli et al. defined the first and second generalized Zagreb indices

$$
\begin{aligned}
M Z_{1}^{a}(G) & =\prod_{u v \in E(G)}\left(d_{u}+d_{u}\right)^{a}, \\
M Z_{2}^{a}(G) & =\prod_{u v \in E(G)}\left(d_{u} \cdot d_{u}\right)^{a} .
\end{aligned}
$$

Multiplicative sum connectivity and multiplicative product connectivity indices [21] are define as:

$$
\begin{gathered}
S C I I(G)=\prod_{u v \in E(G)} \frac{1}{\left(d_{u}+d_{u}\right)}, \\
\operatorname{PCII}(G)(G)=\prod_{u v \in E(G)} \frac{1}{\left(d_{u} \cdot d_{u}\right)} .
\end{gathered}
$$

Multiplicative atomic bond connectivity index and multiplicative Geometric arithmetic index are defined as

$$
\begin{gathered}
A B C I I(G)=\prod_{u v \in E(G)} \sqrt{\frac{d_{u}+d_{u}-2}{d_{u} \cdot d_{u}}}, \\
G A I I(G)=\prod_{u v \in E(G)} \frac{2 \sqrt{d_{u} \cdot d_{u}}}{d_{u}+d_{u}}, \\
G A^{a} I I(G)=\prod_{u v \in E(G)}\left(\frac{2 \sqrt{d_{u} \cdot d_{u}}}{d_{u}+d_{u}}\right)^{a} .
\end{gathered}
$$

In this paper we compute multiplicative indices of circulant graphs.

Definition 1.1. Let $n, m$, and $a_{1}, a_{2}, \ldots, a_{m}$ be positive integers, where $1 \leq a_{i} \leq$ $\left\lfloor\frac{n}{2}\right\rfloor$ and $a_{i} \neq a_{j}$ for all $1 \leq i \leq j \leq m$. An undirected graph with the set of vertices $V=\left\{v_{1}, v_{2}, \ldots, v_{n}\right\}$ and the set of edges $E=\left\{v_{i} v_{i+a_{i}}: 1 \leq i \leq n, 1 \leq\right.$ $j \leq m\}$ where the indices being taken modulo $n$, is called the circulant graph, and is denoted by $C_{n}\left(a_{1}, a_{2}, \ldots, a_{m}\right)$ The graph of $C_{11}(1,2,3)$ is shown in Figure 1. 


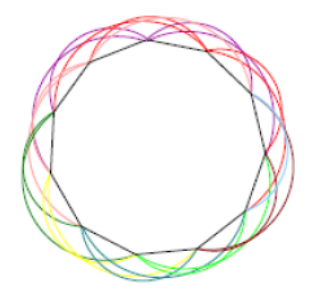

Figure 1. The graph of $C_{11}(1,2,3)$.

This is one of the most comprehensive families, as its specializations give some important families. Classes of graphs that are circulant include the Andr?sfai graphs, antiprism graphs, cocktail party graphs, complete graphs, complete bipartite graphs, crown graphs, empty graphs, rook graphs, M?bius ladders, Paley graphs of prime order, prism graphs, and torus grid graphs.

\section{Computational Results}

In this section, we present our computational results.

Theorem 2.1. Let $C_{n}\left(a_{1}, a_{2}, \ldots, a_{m}\right)$ be the Circulant graph. Then

(1) $M Z_{1}^{a}\left(C_{n}\left(a_{1}, a_{2}, \ldots, a_{m}\right)\right)=2(n-1)^{a n}$,

(2) $M Z_{2}^{a}\left(C_{n}\left(a_{1}, a_{2}, \ldots, a_{m}\right)\right)=(n-1)^{2 a n}$,

(3) $G^{a} A I I\left(C_{n}\left(a_{1}, a_{2}, \ldots, a_{m}\right)\right)=1$.

Proof. Let $C_{n}\left(a_{1}, a_{2}, \ldots, a_{m}\right)$, where $n=3,4, \ldots, n$ and $1 \leq a_{i} \leq\left\lfloor\frac{n}{2}\right\rfloor$ and $a_{i} \neq a_{j}$, when $n$ is odd to be circulant graph. It is clear form the graph of $C_{n}\left(a_{1}, a_{2}, \ldots, a_{m}\right)$ that the circulant gaph has only one partition of the vertex set i.e

$$
V_{1}=\left\{v \in V\left(C_{n}\left(a_{1}, a_{2}, \ldots, a_{m}\right)\right): d_{v}=n\right\} .
$$

The edge partition of $C_{n}\left(a_{1}, a_{2}, \ldots, a_{m}\right)$ has the following partition,

$$
E_{1}=E_{n-1, n-1}=\left\{e=u v \in E\left(C_{n}\left(a_{1}, a_{2}, \ldots, a_{m}\right)\right): d_{u}=d_{v}=n-1\right\} .
$$

Now,

$$
\left|E_{1}\left(C_{n}\left(a_{1}, a_{2}, \ldots, a_{m}\right)\right)\right|=n \text {. }
$$

$$
\begin{aligned}
& M Z_{1}^{a}\left(C_{n}\left(a_{1}, a_{2}, \ldots, a_{m}\right)\right)=\prod_{u v \in E(G)}\left(d_{u}+d_{v}\right)^{a} \\
& =\left(d_{u}+d_{v}\right)^{a\left|E\left(C_{n}\left(a_{1}, a_{2}, \ldots, a_{m}\right)\right)\right|} \\
& =((n-1)+(n-1))^{a n} \\
& =2(n-1)^{a n} \text {. }
\end{aligned}
$$


$(2)$

$$
\begin{aligned}
& M Z_{2}^{a}\left(C_{n}\left(a_{1}, a_{2}, \ldots, a_{m}\right)\right)=\prod_{u v \in E(G)}\left(d_{u} \times d_{v}\right)^{a} \\
& =\left(d_{u}+d_{v}\right)^{a\left|E\left(C_{n}\left(a_{1}, a_{2}, \ldots, a_{m}\right)\right)\right|} \\
& =((n-1) \times(n-1))^{a n} \\
& =(n-1)^{2 a n} \text {. }
\end{aligned}
$$

(3)

$$
\begin{aligned}
G^{a} A I I\left(C_{n}\left(a_{1}, a_{2}, \ldots, a_{m}\right)\right) & =\prod_{u v \in E\left(C_{n}\left(a_{1}, a_{2}, \ldots, a_{m}\right)\right)}\left(\frac{2 \sqrt{d_{u} d_{v}}}{d_{u}+d_{v}}\right)^{a} \\
& =\left(\frac{2 \sqrt{d_{u} d_{v}}}{d_{u}+d_{v}}\right)^{a\left|E_{1}\left(C_{n}\left(a_{1}, a_{2}, \ldots, a_{m}\right)\right)\right|} \\
& =\left(\frac{2 \sqrt{(n-1)^{2}}}{2(n-1)}\right)^{a n} \\
& =1 .
\end{aligned}
$$

Corollary 2.2. Let $C_{n}\left(a_{1}, a_{2}, \ldots, a_{m}\right)$ be the Circulant graph. Then

(1) $M Z_{1}\left(C_{n}\left(a_{1}, a_{2}, \ldots, a_{m}\right)\right)=2(n-1)^{n}$,

(2) $M Z_{2}\left(C_{n}\left(a_{1}, a_{2}, \ldots, a_{m}\right)\right)=(n-1)^{2 n}$,

(3) $\operatorname{GAII}\left(C_{n}\left(a_{1}, a_{2}, \ldots, a_{m}\right)\right)=(1)^{n}=1$.

Proof. We get our result by putting $\alpha=1$ in the Theorem 2.1.

Corollary 2.3. Let $C_{n}\left(a_{1}, a_{2}, \ldots, a_{m}\right)$ be the Circulant graph. Then

(1) $H I I_{1}\left(C_{n}\left(a_{1}, a_{2}, \ldots, a_{m}\right)\right)=2(n-1)^{2 n}$,

(2) $H_{I} I_{2}\left(C_{n}\left(a_{1}, a_{2}, \ldots, a_{m}\right)\right)=(n-1)^{4 n}$,

Proof. We get our desired results by putting $\alpha=2$ in Theorem 2.1.

Corollary 2.4. Let $C_{n}\left(a_{1}, a_{2}, \ldots, a_{m}\right)$ be the Circulant graph. Then

(1) $X I I\left(C_{n}\left(a_{1}, a_{2}, \ldots, a_{m}\right)\right)=\left(\frac{1}{\sqrt{2(n-1)}}\right)^{n}$,

(2) $\chi I I\left(C_{n}\left(a_{1}, a_{2}, \ldots, a_{m}\right)\right)=\left(\frac{1}{n-1}\right)^{n}$.

Proof. We get our desired results by putting $\alpha=\frac{-1}{2}$ in Theorem 2.1.

Corollary 2.5. Let $C_{n}\left(a_{1}, a_{2}, \ldots, a_{m}\right)$ be the Circulant graph. Then

$$
A B C I I\left(C_{n}\left(a_{1}, a_{2}, \ldots, a_{m}\right)\right)=\left(\sqrt{\frac{2(n-2)}{(n-1)^{2}}}\right)^{n} .
$$


Proof. By using the edge partition given in Theorem 2.1, we have

$$
\begin{aligned}
A B C I I\left(C_{n}\left(a_{1}, a_{2}, \ldots, a_{m}\right)\right) & =\prod_{u v \in E\left(C_{n}\left(a_{1}, a_{2}, \ldots, a_{m}\right)\right)} \sqrt{\frac{d_{u}+d_{u}-2}{d_{u} \cdot d_{u}}} \\
& =\left(\sqrt{\frac{(n-1)+(n-2)-2}{(n-1)^{2}}}\right)^{n} \\
& =\left(\sqrt{\frac{2(n-2)}{(n-1)^{2}}}\right)^{n} .
\end{aligned}
$$

\section{Competing Interests}

The authors declare that they have no competing interests.

\section{REFERENCES}

1. Wiener, H. (1947). Structural determination of paraffin boiling points. Journal of the American Chemical Society, 69(1), 17-20.

2. Katritzky, A. R., Jain, R., Lomaka, A., Petrukhin, R., Maran, U., \& Karelson, M. (2001). Perspective on the relationship between melting points and chemical structure. Crystal Growth \& Design, 1(4), 261-265.

3. Rücker, G., \& Rücker, C. (1999). On topological indices, boiling points, and cycloalkanes. Journal of chemical information and computer sciences, 39(5), 788-802.

4. Dobrynin, A. A., Entringer, R., \& Gutman, I. (2001). Wiener index of trees: theory and applications. Acta Applicandae Mathematica, 66(3), 211-249.

5. Du, W., Li, X., \& Shi, Y. (2009). Algorithms and extremal problem on Wiener polarity index. MATCH Commun. Math. Comput. Chem, 62(1), 235.

6. Gutman, I., \& Polansky, O. E. (2012). Mathematical concepts in organic chemistry. Springer Science \& Business Media.

7. Ma, J., Shi, Y., \& Yue, J. (2014). The Wiener Polarity Index of Graph Products. Ars Comb., 116, 235-244.

8. Ma, J., Shi, Y., Wang, Z., \& Yue, J. (2016). On Wiener polarity index of bicyclic networks. Scientific reports, 6, 19066.

9. Gutman, I. (1993). Some properties of the Wiener polynomial. Graph Theory Notes New York, 125, 13-18.

10. Deutsch, E., \& Klavžar, S. (2015). M-polynomial and degree-based topological indices. Iran. J. Math. Chem., (6) 93-102.

11. Gutman, I., \& Trinajstić, N. (1972). Graph theory and molecular orbitals. Total $\phi$-electron energy of alternant hydrocarbons. Chemical Physics Letters, 17(4), 535-538.

12. Gutman, I., Ruščic, B., Trinajstic, N., \& Wilcox Jr, C. F. (1975). Graph theory and molecular orbitals. XII. Acyclic polyenes. The Journal of Chemical Physics, 62(9), 33993405 .

13. Narumi, H., \& Katayama, M. (1984). Simple topological index: A newly devised index characterizing the topological nature of structural isomers of saturated hydrocarbons. Memoirs of the Faculty of Engineering, Hokkaido University, 16(3), 209-214.

14. Gutman, I. (2011). Multiplicative Zagreb indices of trees. Bull. Soc. Math. Banja Luka, 18, 17-23.

15. Todeschini, R., Ballabio, D., \& Consonni, V. (2010). Novel molecular descriptors based on functions of new vertex degrees. Mathematical Chemistry Monographs, 73-100. 
16. Todeschini, R., \& Consonni, V. (2010). New local vertex invariants and molecular descriptors based on functions of the vertex degrees. MATCH Commun. Math. Comput. Chem, 64(2), 359-372.

17. Wang, S., \& Wei, B. (2015). Multiplicative Zagreb indices of k-trees. Discrete Applied Mathematics, 180, 168-175.

18. Eliasi, M., Iranmanesh, A., \& Gutman, I. (2012). Multiplicative versions of first Zagreb index. MATCH Commun. Math. Comput. Chem., 68(1), 217.

19. Kulli, V. R. (2016). Multiplicative hyper-zagreb indices and coindices of graphs: computing these indices of some nanostructures. International Research Journal of Pure Algebra, International Research Journal of Pure Algebra-6(7), 342-347.

20. Kulli, V. R., Stone, B., Wang, S., \& Wei, B. (2017). Generalised multiplicative indices of polycyclic aromatic hydrocarbons and benzenoid systems. Zeitschrift für Naturforschung A, 72(6), 573-576

21. Kulli, V. R. (2016). Multiplicative connectivity indices of $T U C_{4} C_{8}[m, n]$ and $T U C_{4}[m, n]$ nanotubes. Journal of Computer and Mathematical Sciences, 7(11), 599-605.

\section{Ghulam Hussain}

Department of Mathematics, The University of Lahore, Pakpattan Campus, Pakpattan 57400, Pakistan.

e-mail: ghulamhussain151ghs@gmail.com

\section{Saba Noreen}

Department of Mathematics and Statistics, The University of Lahore, Lahore Pakistan.

e-mail: sabaalvi77@yahoo.com

\section{Waseem Khaild}

Department of Mathematics, The University of Lahore, Pakpattan Campus, Pakpattan 57400, Pakistan.

e-mail: waseem.khalid@math.uol.edu.pk 